\title{
Dielectric Behaviour of Some Poly(alkyl methacrylate)s in Dilute Solution
}

\author{
Satoru Mashimo \\ Department of Physics, Faculty of Science, Tokai University, \\ Hiratsuka, Kanagawa, Japan. \\ Akio Chiba \\ Department of Applied Physics, School of Science and Engineering, \\ Waseda University, Shinjuku-ku, Tokyo, Japan.

\section{Kenichi SHINohara} \\ Science and Engineering Research Laboratory, Waseda University, \\ Shinjuku-ku, Tokyo, Japan. \\ (Received October, 12, 1973)
}

\begin{abstract}
The dielectric properties of dilute solutions of PMMA, PEMA and PIBMA in toluene, carbon tetrachloride and dioxane were investigated in the frequency range of $1-150 \mathrm{MHz}$ at temperatures between -23 and $65^{\circ} \mathrm{C}$. A symmetrically shaped absorption curve was observed for each solution. PIBMA exhibits a dielectric behaviour very similar to that of the motion of the main chain. PMMA exhibits a behaviour which can only be interpreted by taking the internal rotation of the side chain into account. PEMA exhibits a behaviour intermediate between PMMA and PIBMA. The dielectric absorption in solution of these polymers can be attributed to the coupled or the cooperative motion of the main chain and the side chain. The dielectric behaviour reflects the magnitude of the two absorptions and manifests a behaviour nearer to that of the larger of the two absorptions.

KEY WORDS Dielectric Behaviour / Dilute Solution / Poly(methyl methacrylate) / Poly(ethyl methacrylate) / Main Chain Motion / Side Chain Motion / Cooperative Motion /
\end{abstract}

It is well-known that polar polymers in solution are divided into two groups according to their dielectric behaviour. ${ }^{1,2}$ The first group exhibits a dielectric absorption in the relatively low frequency region $\left(10^{1}-10^{6} \mathrm{~Hz}\right)$, whose relaxation time is strongly dependent on the molecular weight of the polymer. Poly $(\gamma$-benzylL-glutaminate $)^{3}$ and $\operatorname{poly}(n \text {-buthyl isocyanate })^{4}$ belong to this group. The second group exhibits a dielectric absorption in a rather higher frequency region $\left(10^{6}-10^{12} \mathrm{~Hz}\right)$, and their relaxation time does not depend on the molecular weight of the polymer. Poly(vinyl chloride) ${ }^{5}$ and poly(methyl methacrylate) ${ }^{6,7}$ belong to this group.

When each dipole of a polymer is parallel to the chain direction, the resultant dipole mo- ment as a whole depends on the overall molecular size. When the dielectric absorption is caused by an overall rotation of the molecule as a whole, the relaxation time depends strongly on the molecular weight of the polymer. This is the case for the first group.

Polymers having dipoles rigidly and perpendicularly attached to the chain backbone exhibit molecular-weight-independent relaxation times. Such polymers belong to the second group. In our previous paper, ${ }^{5}$ vinyl polymers such as poly(vinyl chloride), poly( $p$-chlorostyrene), poly ( $p$-bromostyrene) and poly(vinyl acetate) in toluene, carbon tetrachloride and dioxane were investigated in order to clarify the dielectric relaxation mechanism of the second group. The following results were obtained: (1) The relaxa- 
tion time is fairly independent of the molecular weight of the polymer. (2) The shape of the absorption curve is asymmetric, being broader on the high frequency side, irrespective of the kind of polymer, molecular weight of the polymer, solvent, and temperature. (3) The relaxation time is approximately proportional to the solvent viscosity. (4) The apparent activation energy for the dielectric relaxation $\Delta H_{\mathrm{A}}$ depends on that for the solvent viscosity $\Delta H_{\eta}$, in the manner described by $\Delta H_{\mathrm{A}}=\Delta H_{\eta}+C$, where $C$ is a constant. From these results, it was concluded that the dielectric relaxation is attributed to a localized motion of the main chain, and the solvent viscosity plays an important role in reorientating the dipole. By employing the Kramers theory, ${ }^{8}$ it has been proposed that the value of $C$ represents the height of the potential barrier for local conformational change of the main chain.

On the other hand, it has been reported ${ }^{6,7,9}$ that polymers having dipoles attached to flexibles side chains in solution also exhibit a dielectric absorption whose relaxation time does not depend on the molecular weight in the same frequency range as that for vinyl polymers in solution, though the configuration of these polymers are very different. It was therefore thought interesting to investigate whether or not the dielectric relaxation properties of vinyl polymers in solution are the same as those of polymers having dipoles attached to the flexible side chain in solution.

In the present investigation, the dielectric behaviour of poly(alkyl methacrylate)s in solution is discussed in comparison with that of vinyl polymers in solution. The effect of the solvent viscosity on the relaxation time and the activation energy is especially investigated in detail, since vinyl polymers in solution exhibit a dielectric behaviour which is greatly affected by the solvent viscosity. The shape of the absorption curve is also investigated in detail. Poly (methyl methacrylate) (PMMA), poly(ethyl methacrylate) (PEMA) and poly(isobuthyl methacrylate) (PIBMA) were used.

Brouckère, et al., ${ }^{6,7,9}$ performed extensive dielectric measurements on PMMA in toluene, and PNBMA in toluene and carbon tetrachloride in the frequency range of $0.1-35 \mathrm{MHz}$ at temperatures between -30 and $0^{\circ} \mathrm{C}$, and reported that the frequency corresponding to the maximum loss does not depend on the molecular weight of the polymer. However, their measurements were performed at only a few points of temperature, and the value of the activation energy obtained was not sufficiently accurate. Furthermore the shape of the absorption curve and the solvent effect on the relaxation process were not determined. North and Philips ${ }^{10}$ measured dielectric properties of PMMA and PNBMA in toluene in the low temperature region and suggested that the observed dielectric absorption was due to a cooperative motion of the motion of the main chain and the motion of the side chain.

Dielectric measurements on poly(alkyl methacrylate)s in dilute solution in the present investigation were carried out in the frequency range of $1-150 \mathrm{MHz}$ and the temperature range of $-23-65^{\circ} \mathrm{C}$. Solvents used were toluene, carbon tetrachloride and dioxane.

The measurements on PMMA in toluene were carried out, especially, in a wide range of frequency and concentration, i.e., at concentrations of $100-1.6 \%$ and in the frequency range of $30-1.5 \times 10^{8} \mathrm{~Hz}$ in order to investigate the dielectric behaviour of PMMA in dilute solution.

The dielectric relaxation mechanism and the effect of the length of the side chain are discussed also in comparison with those for the solid state.

\section{EXPERIMENTAL}

Polymers used in this investigation were PMMA, PEMA, and PIBMA. PMMA was provided by Fudo Chemical Co., Ltd, and the viscosity-average molecular weight was $1.0 \times 10^{5}$. PEMA was provided by Mitsubishi Rayon Co., Ltd, and PIBMA by Tokyo Kasei Kogyo Co., Ltd. In order to remove monomers and lowmolecular-weight materials contained in these polymers, they were dissolved in toluene and reprecipitated by adding methanol. They were dried in $10^{-3} \mathrm{mmHg}$ over $48 \mathrm{hr}$ at room temperature in order to remove the trace of solvent remaining.

Solvents used were toluene, dioxane, and 
carbon tetrachloride which were dehydrated and distilled before use. The concentration of the solution is expressed by the weight-percent of the polymer.

The method of dielectric measurement in the frequency range of $1-150 \mathrm{MHz}$ was the same as that already reported in the previous papers. ${ }^{5,11}$ The measurements in the frequency range of $3.0 \times 10-1.0 \times 10^{6} \mathrm{~Hz}$ were carried out by using a conventional transformer bridge of Type TR10B manufactured by Ando Electric Co., Ltd.

\section{RESULTS}

Frequency dependences of dielectric constant $\varepsilon^{\prime}$ and loss $\varepsilon^{\prime \prime}$ at various temperatures for PMMA in toluene, PEMA in carbon tetrachloride and PIBMA in dioxane are shown in Figures 1-3. The dispersion curve shows a single inflection and the absorption curve shows a single maximum at any temperature for each polymer solution. The absorption curve is symmetrical.

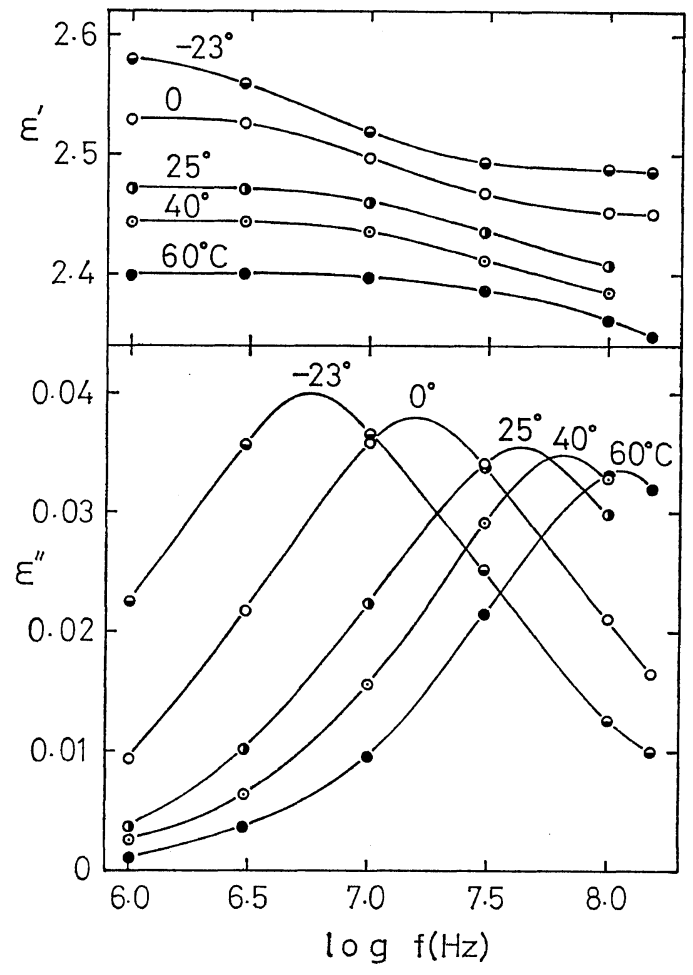

Figure 1. Frequency dependences of dielectric constant and loss at various temperatures for $5.40-\%$ toluene solution of PMMA.
The magnitude of the dielectric absorption could be obtained from the Cole-Cole plot. ${ }^{12}$ The plots are located on a circular arc. The constant $\varepsilon_{0}^{\prime}$ and $\varepsilon_{\infty}^{\prime}$, can then be determined as the intercepts on the abscissa of the Cole-Cole plot, where $\varepsilon_{0}^{\prime}$ and $\varepsilon_{\infty}^{\prime}$ are the low- and highfrequency dielectric constants.

The magnitude of the absorption is plotted against temperature in Figure 4, where $\left(\varepsilon_{0}{ }^{\prime}-\varepsilon_{\infty}{ }^{\prime}\right) / c$ is plotted in place of $\left(\varepsilon_{0}{ }^{\prime}-\varepsilon_{\infty}{ }^{\prime}\right), c$ being the concentration of the polymer. The value of $\left(\varepsilon_{0}^{\prime}-\varepsilon_{\infty}{ }^{\prime}\right) / c$ decreases slightly and linearly with $1 / T$, as temperature rises. This means that the dipole moment remains approximately constant with changing temperature.

The shape of the absorption curve can be represented quantitatively by using Cole's distribution parameter $\beta .^{12}$ Temperature dependence of $\beta$ is shown in Figure 5. The value of $\beta$ changes only slightly with temperature.

In order to determine the apparent activation energy, the logarithm of the frequency, $f_{\mathrm{m}}$, cor-

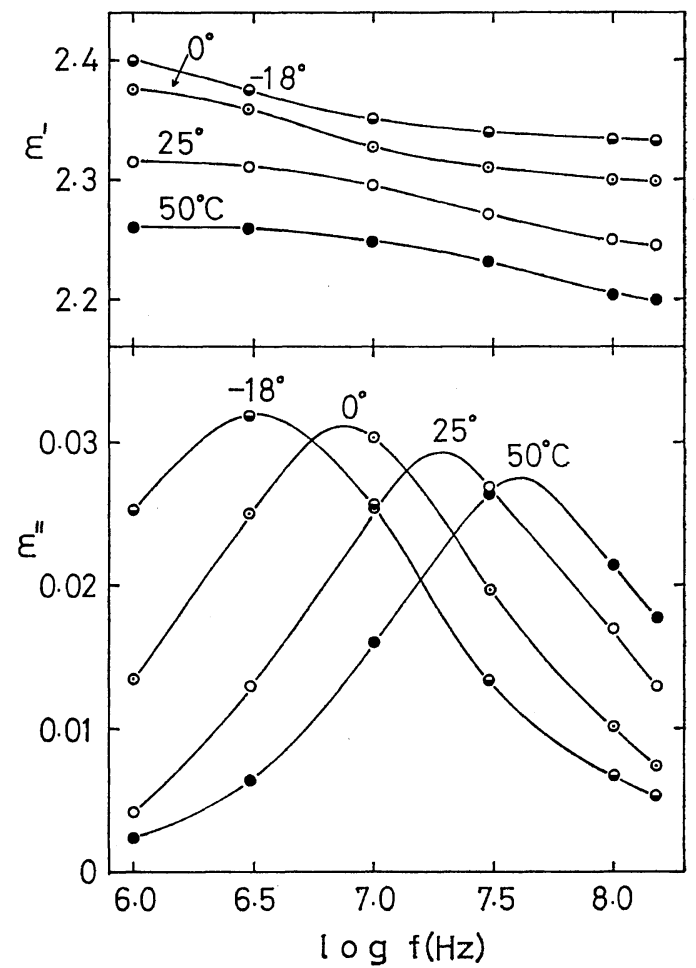

Figure 2. Frequency dependences of dielectric constant and loss at various temperatures for 2.52-\% carbon tetrachloride solution of PEMA.

Polymer J., Vol. 6, No. 2, 1974 
Dielectric Behaviour of Polymers in Solution

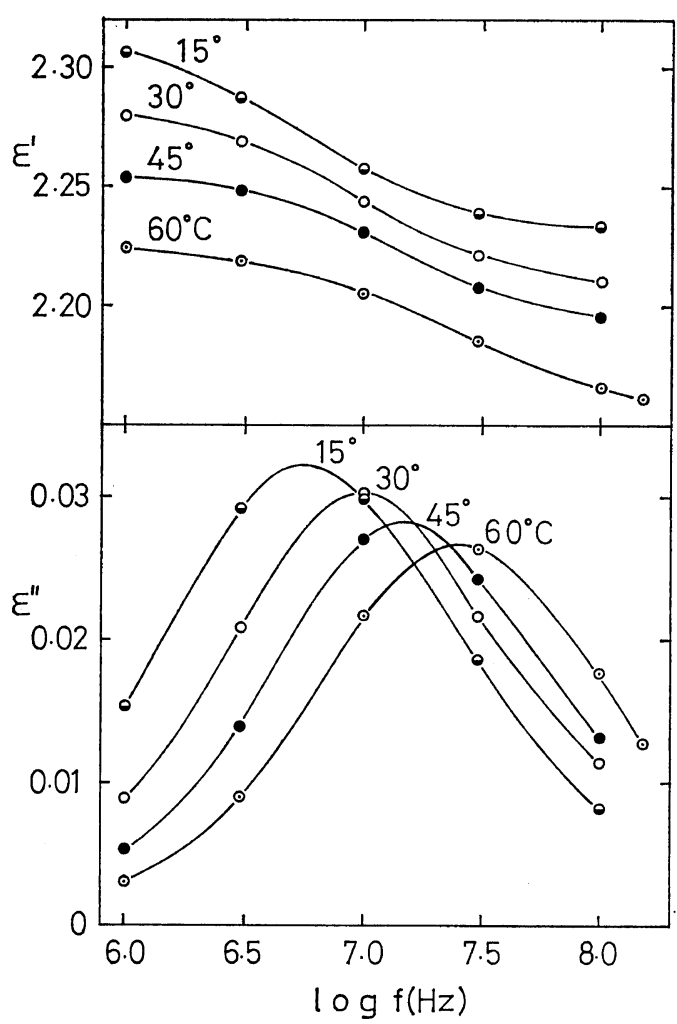

Figure 3. Frequency dependences of dielectric constant and loss at various temperatures for 3.97-\% dioxane solution of PIBMA.

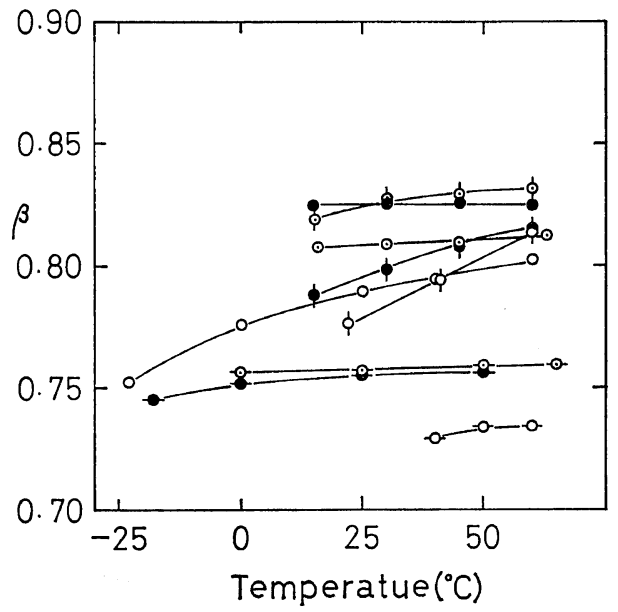

Figure 5. Temperature dependence of $\beta$ for various polymer solutions: Symbols are the same as in Figure 4.

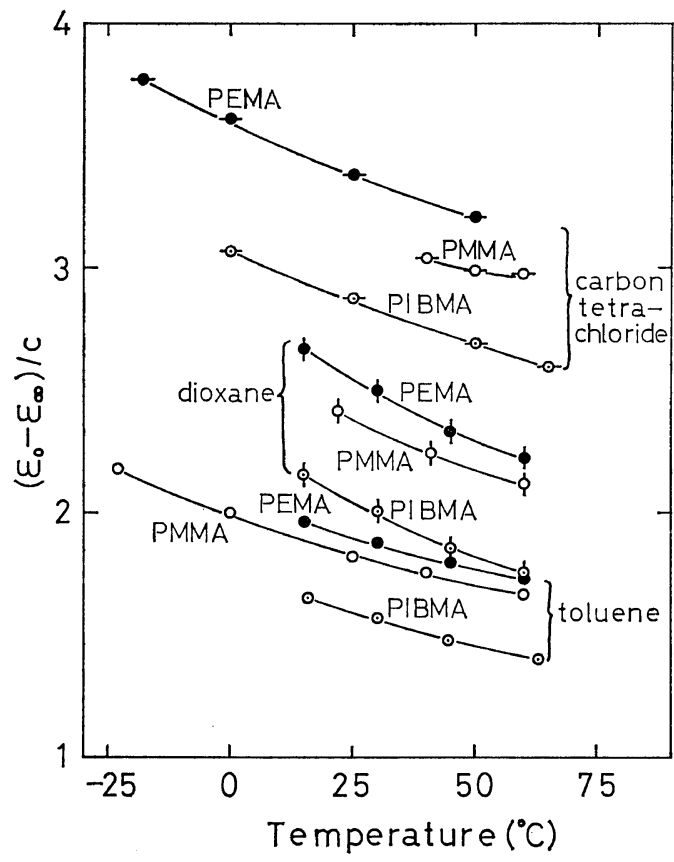

Figure 4. Temperature dependence of $\left(\varepsilon_{0}^{\prime}-\varepsilon_{\infty}\right) / c$ for various polymer solutions: $\bigcirc, 5.40-\%$ PMMA in toluene; - $\bigcirc-, 1.81 \%$ in carbon tetrachloride; $\bigcirc, 2.93 \%$ in dioxane;, $6.49-\%$ PEMA in toluene;

- $2.52 \%$ in carbon tetrachloride;, $3.48 \%$ in dioxane; $\odot, 4.85 \%$ PIBMA in toluene; - - -, 2.08\% in carbon tetrachloride; $\odot, 3.97 \%$ in dioxane.

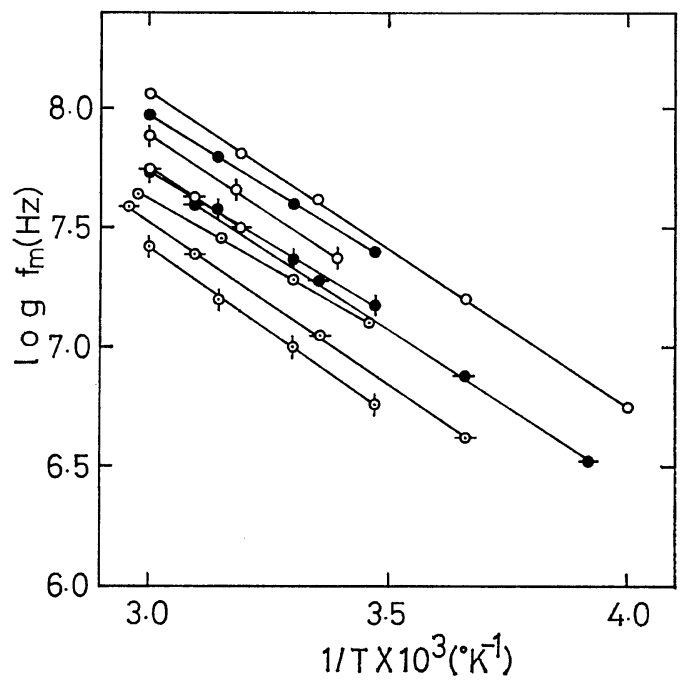

Figure 6. Plot of $\log f_{\mathrm{m}}$ against $1 / T$ for various polymer solutions: Symbols are the same as in Figure 4. 
Table I. Apparent activation energy for various polymer solutions

\begin{tabular}{lccc}
\hline \multirow{2}{*}{ Solvent } & \multicolumn{3}{c}{$\Delta H_{\mathrm{A}}, \mathrm{kcal} / \mathrm{mol}$} \\
\cline { 2 - 4 } Polymer & Toluene & $\begin{array}{c}\text { Carbon } \\
\text { tetrachloride }\end{array}$ & Dioxane \\
\hline PMMA & 6.0 & 6.0 & 6.0 \\
PEMA & 5.6 & 5.9 & 5.6 \\
PIBMA & 5.2 & 6.1 & 6.4 \\
$\Delta H_{\eta}$ & 2.1 & 2.6 & 3.0 \\
\hline
\end{tabular}

Table II. Concentration dependences of dielectric relaxation parameters for each polymer in toluene solution

\begin{tabular}{ccccccc}
\hline \multicolumn{2}{c}{$\begin{array}{c}\text { Temp, Concn, } \\
{ }^{\circ} \mathrm{C}\end{array}$} & $\begin{array}{l}\log f_{\mathrm{m}}, \\
\mathrm{Hz}\end{array}$ & $\left(\varepsilon_{0}^{\prime}-\varepsilon_{\infty}\right) / c$ & $\beta$ & $\begin{array}{c}\left(\mu^{2} / z\right)^{1 / 2} \\
D\end{array}$ \\
\hline PMMA 60 & 1.60 & 8.08 & 1.66 & 0.80 & \\
& & 5.40 & 8.06 & 1.67 & 0.80 & 1.33 \\
PEMA 45 & 4.16 & 7.80 & 1.80 & 0.81 & \\
& & 6.49 & 7.80 & 1.80 & 0.81 & 1.43 \\
PIBMA 44.5 & 1.90 & 7.47 & 1.43 & 0.81 & \\
& 2.97 & 7.46 & 1.45 & 0.81 & \\
& 4.85 & 7.46 & 1.47 & 0.81 & 1.41 \\
\hline
\end{tabular}

responding to the maximum loss is plotted against the reciprocal of the absolute temperature as shown in Figure 6. For each solution the plot of $\log f_{\mathrm{m}}-1 / T$ gives a straight line. The apparent activation energy $\left(\Delta H_{\mathrm{A}}\right)$ obtained in this way for each solution is listed in Table I.

In order to investigate the effect of the concentration on the dielectric behaviour, toluene solution was employed and the concentration of PMMA, PEMA, or PIBMA was varied from about 6 to $1 \%$. The values of $\log f_{\mathrm{m}}, \beta$, and $\left(\varepsilon_{0}{ }^{\prime}-\varepsilon_{\infty}{ }^{\prime}\right) / c$ at various concentrations are listed in Table II. Since the value of $f_{\mathrm{m}}$ and $\beta$ are not affected by the concentration, the value of $\beta$ shown in Figure 5 and the value of $\Delta H_{A}$ in Table I may also be valid for a solution of very low concentration or even one of zero concentration.

Dipole moments for a repeat unit $\left(\mu^{2} / z\right)^{1 / 2}$ were calculated by using Onsager's equation ${ }^{13,11}$ and extrapolating the quantities appearing in the equation to zero concentration, where $z$ is the degree of polymerization. The value of $\left(\mu^{2} / z\right)^{1 / 2}$ is also given in Table II. The dipole moment for a repeat unit of PMMA is about
Table III. Ratio of relaxation time to solvent viscosity

\begin{tabular}{|c|c|c|c|c|c|}
\hline \multirow{2}{*}{\multicolumn{2}{|c|}{$\underset{{ }^{\circ} \mathrm{C}}{\text { Temp }}$}} & \multirow{2}{*}{$\begin{array}{l}\left(\tau / \eta_{0}\right)_{\text {mean }} \\
\text { sec } \text { poise }^{-1}\end{array}$} & \multicolumn{3}{|c|}{$\left(\tau / \eta_{0}\right) /\left(\tau / \eta_{0}\right)_{\text {mean }}$} \\
\hline & & & $T^{\mathrm{a}}$ & $C . T^{\mathrm{a}}$ & $D^{\mathrm{a}}$ \\
\hline \multirow[t]{3}{*}{ PMMA } & 60 & $3.90 \times 10^{-7}$ & 0.93 & 1.23 & 0.78 \\
\hline & 45 & 5.06 & 0.94 & 1.25 & 0.74 \\
\hline & 30 & 6.77 & 0.96 & 1.25 & 0.72 \\
\hline \multirow[t]{4}{*}{ PEMA } & 60 & $4.66 \times 10^{-7}$ & 0.96 & 1.10 & 0.86 \\
\hline & 45 & 5.98 & 0.98 & 1.14 & 0.86 \\
\hline & 30 & 7.71 & 0.99 & 1.15 & 0.83 \\
\hline & 15 & 10.0 & 1.01 & 1.15 & 0.77 \\
\hline \multirow[t]{4}{*}{ PIBMA } & 60 & $0.98 \times 10^{-6}$ & 1.11 & 0.90 & 0.98 \\
\hline & 45 & 1.16 & 1.09 & 0.93 & 0.97 \\
\hline & 30 & 1.52 & 1.05 & 0.97 & 0.99 \\
\hline & 15 & 2.03 & 1.03 & 1.00 & 0.98 \\
\hline
\end{tabular}

$0.1 D$ smaller than that of PEMA or PIBMA.

The value of $\Delta H_{\mathrm{A}}$ obtained for PMMA or for PEMA in dilute solution is about $6.0 \mathrm{kcal} / \mathrm{mol}$ and is independent of the solvent. On the other hand, the value for PIBMA in dilute solution is affected by the solvent. The dependence of the activation energy for the dielectric relaxation process on the activation energy for the solvent viscosity for PIBMA in solution is very similar to that of vinyl polymer in solution.

In order to estimate the effect of the solvent viscosity on the relaxation time $\tau\left(=1 /\left(2 \pi f_{\mathrm{m}}\right)\right)$, the mean root square value of $\tau / \eta_{0}$ was estimated for each polymer solution, where $\eta_{0}$ is the solvent viscosity. The values of $\left(\tau / \eta_{0}\right) /\left(\tau / \eta_{0}\right)_{\text {mean }}$ was, then, calculated (Table III). In this estimation the value of $f_{\mathrm{m}}$ was obtained from the plot of $\log f_{\mathrm{m}}-1 / T$ at temperatures of $60,45,30$, and $15^{\circ} \mathrm{C}$. For PMMA, the value of $\left(\tau / \eta_{0}\right) /\left(\tau / \eta_{0}\right)_{\text {mean }}$ deviates from unity for various solvents, but for PIBMA it is approximately a constant and is close to 1 , meaning that the relaxation time is proportional to the solvent viscosity. Table III also shows that when the side chain is longer, the deviation of the value of $\left(\tau / \eta_{0}\right) /\left(\tau / \eta_{0}\right)_{\text {mean }}$ from unity is smaller.

Measurement of the dielectric dispersion and absorption on PMMA-toluene system was carried out at a wide range of concentrations of $100-1.6 \%$ at a temperature of $60^{\circ} \mathrm{C}$. The frequency dependence of the dielectric loss ob- 


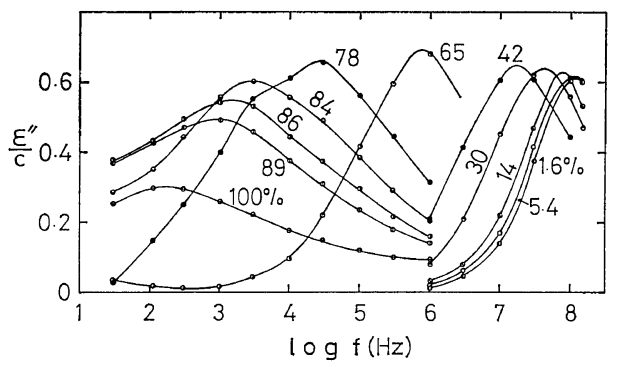

Figure 7. Frequency dependences of dielectric loss for PMMA-toluene system at $60^{\circ} \mathrm{C}$.

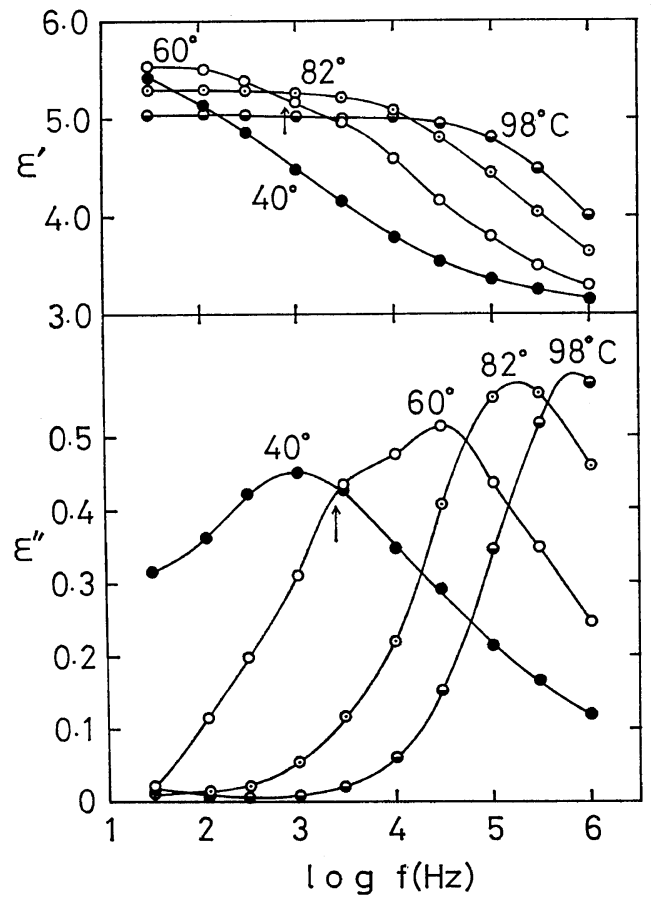

Figure 8. Frequency dependences of dielectric constant and loss at various temperatures for PMMA-toluene system (78-\% PMMA).

tained is shown in Figure 7. At concentrations above $84 \%$, only one absorption peak is observed in each curve in the frequency range measured. At $78 \%$, another small absorption is observed. Furthermore, at concentrations below $68 \%$, this small peak merges into the former absorption, and becomes indistinguishable in the solution of lower concentration. This small absorption is reflected also in dispersion curve shown in Figure 8 and is indicated by

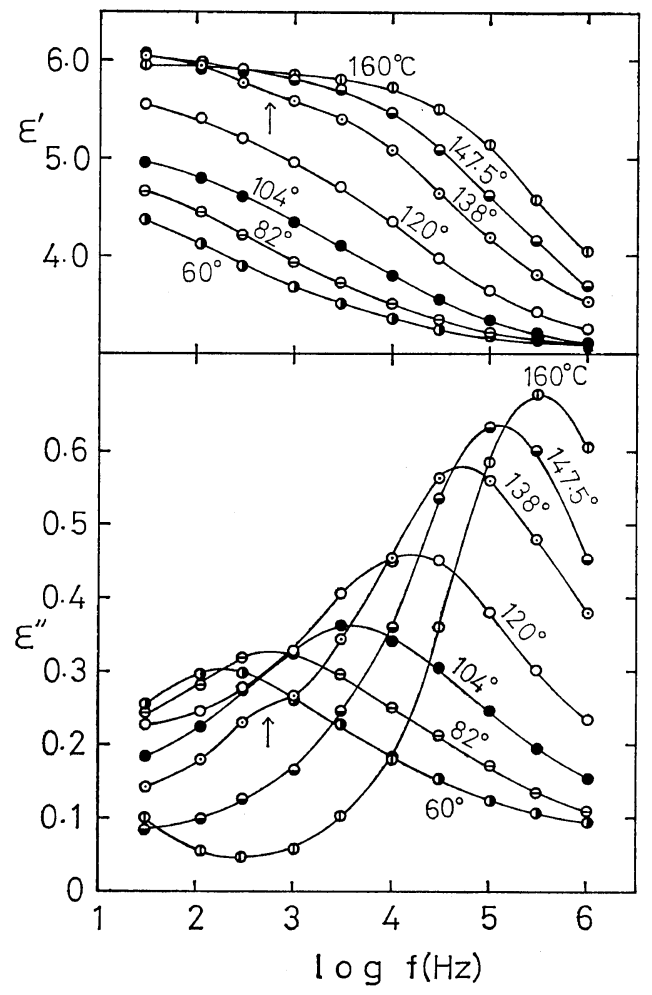

Figure 9. Frequency dependences of dielectric constant and loss at various temperatures for solid PMMA.

an arrow.

In the solid PMMA, only one absorption peak is observed at low temperatures, but at $138^{\circ} \mathrm{C}$ another small peak makes its appearance in the usual range of frequency measurement, which merges into the former absorption peak at higher temperatures (Figure 9). It was reported that the latter small peak is attributed to the motion of the main chain and the former peak to the motion of the side chain. ${ }^{14,16}$ Lowering the concentration in solution, thus, gives an effect on the frequency dependence of the absorption curve similar to that of raising the temperature in the case of a solid.

\section{DISCUSSION}

The results obtained show that PMMA in solution exhibits quite different dielectric properties from those of vinyl polymers in solution. The differences observed are in the effect of the 
solvent viscosity of the activation energy and the relaxation time, and also in the shape of the absorption curve. The activation energy of PMMA in solution is always $6.0 \mathrm{kcal} / \mathrm{mol}$ for any solvent used. The ratio of the relaxation time to the solvent viscosity is not constant and the shape of the absorption curve is symmetrical. These observations indicate that the relaxation mechanism for PMMA in solution is not the same as that for the vinyl polymers in solution.

In the case of PEMA in solution, the relaxation time is approximately proportional to the solvent viscosity, i.e., within $\pm 20 \%$. However, the activation energy is $5.6-5.9 \mathrm{kcal} / \mathrm{mol}$ and does not depend much on the solvent, the shape of the absorption curve is symmetrical.

In the case of PIBMA in solution, the activation energy was found to depend on the solvent and the relaxation time was proportional to the solvent viscosity within $\pm 10 \%$. The dependences of the activation energy and the relaxation time on the solvent are very similar to those for vinyl polymers in solution. A difference in the dielectric property between the PIBMA in solution and the vinyl polymers in solution is observed only in the shape of the absorption curve. PIBMA shows a symmetrical curve, whereas the vinyl polymers show an asymmetric curve. Thus, the length of the side chain gives quite an important effect on the dielectric behaviour of poly(alkyl methacrylate)s in solution.

In solid poly(alkyl methacrylate)s too, the length of the side chain has a great influence on the dielectric behaviour. ${ }^{14-16}$ In the case of PMMA the magnitude of the dielectric absorption due to the internal rotation of the side chain is very large compared to that due to the motion of the main chain. ${ }^{14-18}$ The ratio of the former to the latter is about 3 at $100^{\circ} \mathrm{C}$, and it takes still larger values at higher temperatures. $^{14}$ For PEMA the two are comparable. ${ }^{14-16,18}$ When the side chain is longer than that of PEMA the ratio takes a value less than unity. ${ }^{14-16,18}$

It is pointed out above that dielectric absorption is a dilute solution of PMMA closely corresponds to that in the solid state at high temperatures. Therefore if the magnitude of dielectric absorption due to the internal rotation of the side chain remains far larger than that due to the motion of the main chain even at high temperatures in the solid state, the former would also be far larger than the latter in the dilute solution. When the dielectric absorption in caused mainly by the internal rotation of the side chain, an adequate explanation can be given for the dielectric behaviour of PMMA in solution. The relaxation time in such a case does not depend on the solvent viscosity, and the activation energy represents the height of the potential barrier for the internal rotation and will not depend on the solvent viscosity.

Similar arguments will lead to the conclusion that for PEMA in solution the magnitude of the dielectric absorption due to the motion of the main chain may be comparable to that due to the internal rotation of the side chain and the dielectric behaviour may show an behaviour intermediate between that for the motion of the main chain and for the internal rotation of the side chain. For PIBMA in solution the magnitude of the absorption due to the motion of the main chain may be far larger than that of the side chain and the dielectric behaviour shows the same feature as that for the motion of the main chain.

Comparison of the magnitude of the absorption due to the motion of the main chain and that of the internal rotation of the side chain alone cannot, however, describe all of the experimental results. It was shown above, by an experiment in which the solvent viscosity was varied, that some kind of cooperation exists between those two kinds of motions. If the two motions are independent, we can separate the absorption due to the two motions into two absorptions due to each motion using solvents of varying viscosity, since the motion of the main chain is greatly affected by the solvent viscosity but the internal rotation of the side chain may be little affected by it. This was found not to be the case. The existence of the cooperative motion was first introduced by Williams to solid PEMA and solid PNBMA. ${ }^{19,20}$

According to Williams, the reorientation of the dipole component perpendicular to the chain backbone and that of the dipole component perpendicular to the above component are inter- 
correlated at high temperatures, and the absorptions due to the reorientation of the two components are not separable. On the other hand, the movement of the former component will involve a small scale movement of the main chain as temperature rises and at high temperature the absorption due to this motion cannot be distinguishable from the absorption due to the motion of the latter component. Further, the two dipole components move together as a single unit at this temperature.

As far as the fact that the absorption due to the motion of the main chain and that of the side chain could not be resolved, this notion of cooperative motion seems to be valid also for poly(alkyl methacrylate)s in solution.

The experimental results in dilute solution indicate that the cooperative motion does not give rise to the same dielectric behaviour for all kinds of poly(alkyl methacrylate)s in solution. PMMA in solution exhibits a dielectric behaviour which is interpreted by taking into account only the internal rotation of the side chain, PIBMA in solution exhibits a dielectric behaviour ascribable to the motion of the main chain and PEMA in solution exhibits a behaviour intermediate between PMMA and PIBMA in solution. These results lead either to the conclusion that the cooperative motion is affected to a great extent by the length of the side chain, or the conclusion that the dielectric behaviour is determined by the relative magnitude of the absorption due to the motion of the main chain and that due to the motion of the side chain at the temperature at which the two motions do couple. In consequence, when the magnitude of the dielectric absorption due to the motion of the main chain is far larger than that due to the internal rotation of the side chain, the observed dielectric behaviour will be similar to that of the motion of the main chain. This is the case for the dielectric behaviour of PIBMA in solution. Dielectric behaviour of poly(vinyl acetate) in solution also corresponds to this case. ${ }^{5}$ In the converse case, the observed dielectric behaviour will exhibit the dielectric behaviour of internal rotation of the side chain. The dielectric behaviour of PMMA in solution belongs to this case.

Polymer J., Vol. 6, No. 2, 1974

\section{CONCLUSION}

Dielectric behaviour of poly(alkyl methacrylate)s in dilute solution is largely affected by the length of the side chain. In a solution of PMMA which has the shortest side chain, the dielectric behaviour represents features arising from the internal rotation of the side chain. In a solution of PIBMA whose side chain is rather long, the dielectric behaviour resembles that given by the motion of the main chain. In a solution of PEMA whose side chain is of an intermediate length between PMMA and PIBMA, the dielectric behaviour exhibits an behaviour intermediate between those of PMMA and PIBMA. The observed dielectric behaviour is interpreted as being due to the coupled or the cooperative motion of the main chain and the side chain motions, and its main feature is obtained by comparing the magnitude of the two absorptions, one due to the motion of the main chain and the other due to that of the internal rotation of the side chain. The dielectric behaviour reflects that of the larger absorption between those two absorptions.

\section{REFERENCES}

1. W. H. Stockmayer, Pure and Appl. Chem., 15, 539 (1967).

2. T. Bates, K. J. Ivin, and G. Williams, Trans. Faraday Soc., 63, 1964 (1967).

3. A. Wada, J. Chem. Phys., 29, 674 (1958); ibid., 31, 495 (1959).

4. H. Yu, A. J. Bur, and L. J. Fetters, ibid., 44, 2568 (1966).

5. S. Mashimo and A. Chiba, Polymer J., 5, 41 (1973).

6. L. de Brouckère, D. Buess, J. de Bock, and J. Versluys, Bull. Soc. Chim. Belg., 64, 669 (1955).

7. L. de Brouckère, D. Buess, and L.K. H. van Beek, J. Polym. Sci., 23, 233 (1957).

8. E. Helfand, J. Chem. Phys., 54, 4651 (1971).

9. N. Hill, W.E. Vaughan, A.H. Price, and M. Davies, "Dielectric Properties \& Molecular Behaviour," Van Nostrand Reinhold Company, London, 1969, pp 364-365.

10. A. M. North and P. J. Philips, Trans. Faraday Soc., 63, 1537 (1967).

11. S. Mashimo and K. Shinohara, J. Phys. Soc. Japan, 34, 1141 (1973). 
S. Mashimo, A. Chiba, and K. Shinohara

12. K. S. Cole and R. H. Cole, J. Chem. Phys., 9, 341 (1941).

13. L. Onsager, J. Amer. Chem. Soc., 58, 1486 (1936).

14. Y. Ishida and K. Yamafuji, Kolloid-Z., 177, 97 (1961).

15. J. Heijboer, Makromol. Chem., 35A, 86 (1960).

16. G. P. Mikhailov, J. Polym. Sci., 30, 605 (1958).

17. S. Saito and T. Nakajima, J. Appl. Polym.
Sci., 2, 93 (1959).

18. M. G. McCrum, B. E. Read, and G. Williams, "Anelastic and Dielectric Effects in Polymeric Solid," John Willy \& Sons, Inc., New York, N.Y., 1967, Chapter 8, pp 257-271.

19. G. Williams, Trans. Faraday Soc., 62, 2091 (1966).

20. G. Williams and D. A. Edwards, ibid., 62, 1329 (1966). 